\title{
Effects of the Menstrual Cycle on Language and Visual Working Memory: A Pilot Study
}

\author{
Kiyomi KONISHI ${ }^{1 *}$, Masaharu KUMASHIRO², Hiroyuki IZUMI², \\ Yoshiyuki HIGUCHI ${ }^{3}$ and Yayoi $\mathrm{AWA}^{1}$
}

\author{
${ }^{1}$ Department of Sciences in Nursing, Faculty of Human Health Sciences, Meio University, 1220-1 Bimata, \\ Nago city, Okinawa 905-8585, Japan \\ ${ }^{2}$ Department of Ergonomics, Institute of Industrial Ecological Science, University of Occupational and \\ Environmental Health, 1-1 Iseigaoka, Yahatanishi-ku, Kitakyushu 807-8555, Japan \\ ${ }^{3}$ Department of faculty of Nursing, Fukuoka Prefectural University, 4395 Ita, Tagawa, Fukuoka 825-8585, \\ Japan
}

Received November 20, 2008 and accepted March 30, 2009

\begin{abstract}
This study aimed to examine the effects on language and visual working memory of the follicle, luteal, and menstruation phases. The subjects were 12 female students. We added visual information and the assignment of experimental tasks, which requires the faculty of sight, to the previous study; therefore, this study has an index of visual and linguistic working memory. Computer-based tasks formulated by the authors, using the working memory that actively retains the information as the index, were used for experiments of $\mathbf{3 0}$ min during the follicle, luteal, and menstruation phases. After completion of the experiment of each phase, blood samples were collected in order to clearly distinguish the three phases of the subjects' menstrual cycle, and the session order was counter-balanced. The results showed that mental workload was higher in the luteal phase and stress was more easily felt. Indefinite complaints were most frequent during the menstruation phase, but task performance was good regardless of the indefinite complaint. It suggested that when the same tasks were performed during three phases, the sympathetic nerve was ascendant.
\end{abstract}

Key words: Menstrual cycle, Working memory, Performance, Sex steroid hormones, Premenstrual symptoms

\section{Introduction}

Nowadays Japanese women are remarkably active in society, and the areas in which they work are expanding.Also the positive action for ability display of working women is promoted in many companies ${ }^{1)}$. However, women have physical and mental symptoms during the menstrual cycle, and it is also said that $80-90 \%$ of women are aware of their indefinite menstrual complaints ${ }^{2}$.

In today's nursing profession, in which many women are employed, women must cope with high technology and informed consent, such as the introduction of the electronic chart, and the complex and diverse needs of

*To whom correspondence should be addressed.

E-mail: konishi@meio-u.ac.jp patients. Under these circumstances, women have to prioritize plural work and they should accomplish the tasks efficiently. When they carry out the tasks effectively, the work of the working memory plays an important role. With the work of the working memory, which was advocated by Baddeley ${ }^{3}$, based on the model that I proposed, there is a spatial sketchpad system and three subsystems of the episode buffer of the central executive system that is a control structure of attention, the phonological loop, and sight. This consists of visual meaning and language interpretation and the data storehouses such as an episode or long-term memory. There are two views with regard to the functioning of working memory. One emphasizes the active retention of information for a certain period of time; the other emphasizes the flexible processing of the actively retained information ${ }^{4)}$. 
In our antecedent study, we reported ${ }^{5)}$ an estrous cycle in an object of the time of 2 of the follicle phase and the luteal phase among the models of the working memory particularly of the phonological loop worked, in other words, the subjects were made to conduct tasks that focused on keeping information active for tasks requiring language working memory. The results showed that there was a significantly lower error rate for working memory tasks in the premenstrual phase, suggesting that task performance was good. Mild premenstrual symptoms had no effect on working memory function of the premenstrual phase and the estrogen sex hormone secreted from the ovaries in relation to the menstrual cycle is thought to be involved in the working memory function.

In the precedent study, as for the problems, such as fluency characteristics or careful handwork (continuous handwork test) of the language, estrogen had particularly good results in an estrous cycle in a luteal phase for the high density period ${ }^{6,7)}$. Meanwhile, no significant differences were seen between the control group and the PMDD (premenstrual dysphoric disorder) group in the context of Spatial Working Memory; however, it has been reported that a significantly higher error rate was found in both groups for the premenstrual phase as compared to the postmenstrual phase ${ }^{8)}$. Moreover, it was also found that the high performance of the language working memory is likely related to the highly concentrated estrogen level ${ }^{9)}$. Not much is known due to the fact that in previous studies the ages of the subjects varied and different job characteristics were measured. Further, a uniform viewpoint could not be obtained in regards in which phase of the menstrual cycle working memory function deteriorates.

We measured the sex steroidal hormone level at this time and determined the menstruation period clears in the luteal phase for the follicle period and clearly distinguished the menstrual cycle. In addition, we created tasks that focused on the processing of information kept active using the phonological loop of Baddeley ${ }^{3)}$ and a sight and special sketchpad system as models and used this as a working memory index. We added a task for sight to a working memory task for language that we reported the first time and made this index as a multiplex task. In other words, of the entire menstrual cycle, it is thought that for visual and language working memory, which is a multiplex task, the results may worsen in the luteal phase depending on the task characteristics. Thus, using language and visual working memory as the index, this study aimed to gain fundamental knowledge for the purpose of managing women's occupational health and safety, by examining task performance capability from both subjective and physical aspects in terms of the effect on working memory of three phases of the menstrual cycle: the follicle, luteal, and menstruation phases.

\section{Subjects and Methods}

\section{Subjects}

The subjects were 12 healthy female students majoring in nursing at university $\mathrm{O}$, with an average age of $22.4 \pm 1.1$, and the student nurses are not actively practicing yet. The subjects normal vision or corrected vision in both eyes of average $1.1 \pm 0.3$, their average computer experience was $5.2 \pm 1.8 \mathrm{yr}$, and all were right-handed. The subjects, who were selected, gave their consent after the aim and methods of this study were described to them. Those selected also recorded their basal body temperature for three months, had regular menstrual cycles, biphasic body temperature, were not taking hormones, oral contraceptives or any nonprescription drugs, and had never conceived a child. The average menstrual cycle of the subjects was $31.7 \pm 3.5 \mathrm{~d}$.

They were instructed to get sufficient rest and avoid alcohol intake the day before the experiment, not to consume caffeine on the day of the experiment, and to avoid eating, drinking or going to the toilet less than $30 \mathrm{~min}$ before the experiment. Two of the subjects who stopped the experiment partway through were excluded from consideration.

\section{Procedure}

The experiment took place in individual rooms with ambient silence, between the hours of $2 \mathrm{pm}$ and $6 \mathrm{pm}$. The temperature was $26.42 \pm 0.57^{\circ} \mathrm{C}$ and humidity was $56.83 \pm 8.25 \%$. For this study, measurement of task performance was carried out three times, during the follicle phase, luteal phase, and menstruation phase, respectively. In order to avoid the order effect during the three phases, the experiment was counterbalanced by having four women start during the follicle phase, four during the luteal phase, and four during the menstruation phase. The subjects filled in general information, answered and the self-recorded MDQ survey, and then were fitted with portable electrocardiographs (ECG) before the experiment. After resting for $10 \mathrm{~min}$, they practiced the tasks for $2 \mathrm{~min}$, then rested for $1 \mathrm{~min}$, then carried out the tasks for $30 \mathrm{~min}$. After completing the tasks they rested for 10 min, and then answered the NASA-TLX.

The subjects were instructed to do the experiment tasks by clicking as quickly and accurately as possible, and to keep their eyes lightly closed during the rest phase before and after the experiment. After completion of the experiment, blood samples were collected in order to clearly distinguish the three phases of the menstrual cycle. 

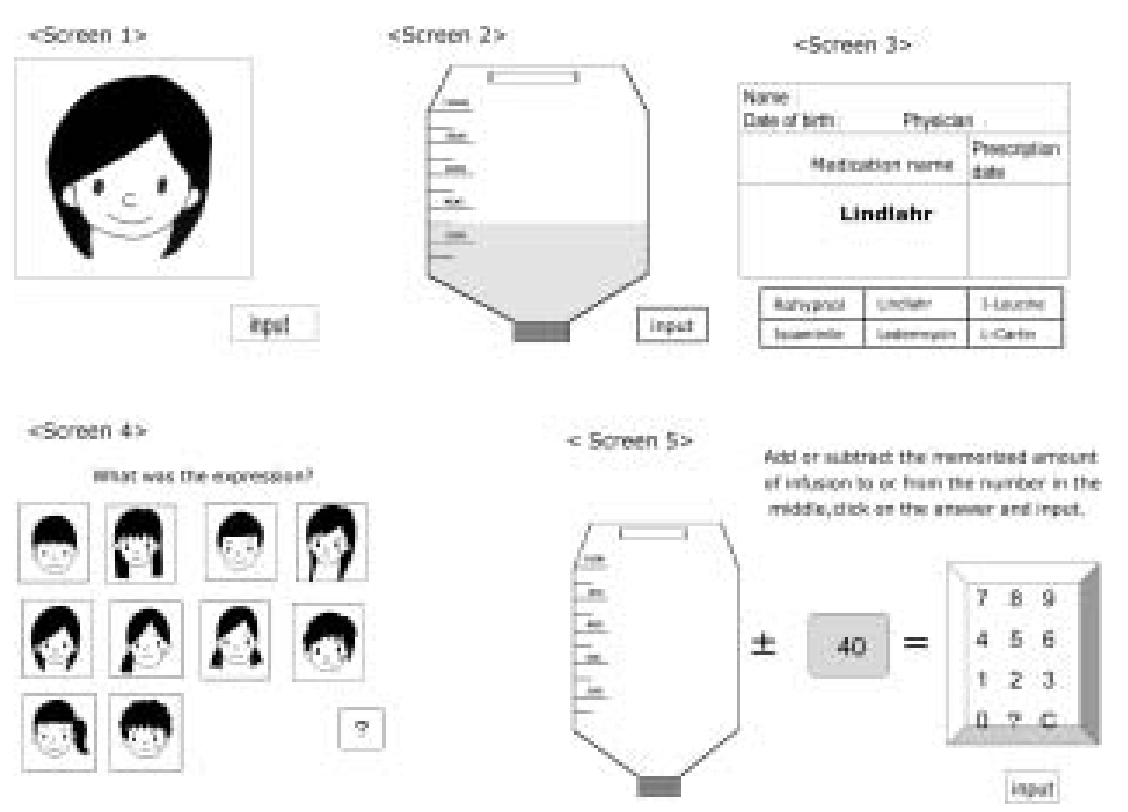

Fig. 1. Experiment task: work memory index.

Screen 1: The patient is memorized. Screen 2: The amount of medication on screen 2 is memorized. Screen 3: The task is carried out for $10 \mathrm{~s}$. Screen 4: The patient memorized on screen 1 is selected. Screen 5: A two-digit integer is calculated mentally from the number memorized on screen 2 and the facial expression of the patient on screen 1 .

\section{Experimental tasks}

Visual information on the task reported ${ }^{5,10)}$ earlier based on Baddely ${ }^{3)}$ and a working model was added to the experiment problem of this study. That simulates "medication preparation work" that is part of the representative work of nurses. The experiment tasks for this study were carried out using a mouse on a mouse pad $(140 \times 180 \mathrm{~mm})$ to click on a computer (Dell Sharp 17" PC) for $30 \mathrm{~min}$ (Fig. 1). The first task entailed clicking on OK once the amount in the medication bottle on the first screen was memorized. The primary task was to memorize the picture ("patient") (screen 1) and the amount of medication (screen 2). Then the screen changed for the secondary task, where names of medications were checked for $10 \mathrm{~s}$ (screen 3 ), and then the patient memorized for the primary task had to be selected (screen 4) and depending on the patient's facial expression, a two-digit number had to be added to or subtracted from the memorized amount of medication (screen 5). This task was evaluated by the percentages of error which indicated the ability of the subjects to maintain visual and linguistic information using the working memory as its index.

Screen 1: A patient is displayed randomly. The patient's expression, smiling or crying, is memorized. "Input" is clicked after memorization.

Screen 2: The amount of medication is displayed randomly, in a bottle marked at $100 \mathrm{ml}$ intervals from 100 to $1,000 \mathrm{ml}$. "Input" is clicked after the amount is memorized.

Screen 3: A prescription with the name of a medication consisting of six letters is shown, and the same name must be selected from among the six types of medications shown below. When one answer is clicked, a different prescription appears, and this task is carried out for ten seconds.

Screen 4: Ten male and female patients are displayed, five of whom are smiling and five of whom have tearful faces. The patient memorized on screen 1 is selected from among these.

Screen 5: Depending on the patient's expression, a twodigit integer is randomly displayed relative to the amount of infusion memorized on screen 2. If the patient's expression memorized on screen 1 was smiling, then that two-digit integer is added "+", while if the expression was tearful it is subtracted " - " and that result is input. If the subjects forgot or did not know, they input "?", and "C" was selected for an input error.

The above tasks on screens 1-5 were carried out repeatedly for $30 \mathrm{~min}$. The subjects were instructed to carry out the experiment tasks by clicking as quickly and as accurately as possible, and to keep their eyes lightly closed during the post-experiment rest period. 
Table 1. Working memory task results in the menstrual cycle $(n=12)$

\begin{tabular}{lccc}
\hline & Follicle phase & Luteal phase & Menstruation phase \\
\cline { 2 - 4 } & Mean (SD) & Mean (SD) & Mean (SD) \\
\hline Primary task & & & \\
Number of tasks completed & $81.67(12.06)$ & $77.42(9.45)$ & $81.75(9.15)$ \\
1)Patient Error rate (\%) & $8.81(6.58)$ & $11.25(9.04)$ & $8.26(5.47)$ \\
${ }^{2)}$ Task Error rate (\%) & $10.59(7.24)$ & $16.69(12.21)$ & $10.33(7.95)$ \\
& \multicolumn{3}{c}{$*$} \\
\hline Secondary task & \multicolumn{3}{|c}{} \\
$\quad$ Number of confirmations & $403.58(103.45)$ & $369.25(72.99)$ & $402.92(86.61)$ \\
${ }^{3}$ Error rate $(\%)$ & $1.66(1.71)$ & $1.85(1.69)$ & $1.70(2.09)$ \\
\hline
\end{tabular}

1) $(1-$ Number of correct patient answers/Number of tasks completed $) \times 100$.

2) $(1-$ Number of correct task answers/Number of tasks completed $) \times 100$.

3) $(1-$ Number of correct answers/Number of confirmations $) \times 100$.

$* p<0.05$ : Bonferroni test.

\section{Subjective indices $M D Q$}

In order to assess the physical and mental state in the menstrual cycle, the applied questionnaire was the MDQ (Menstrual Distress Questionnaire), developed in order to gain an understanding of indefinite menstrual complaints, i.e., physical and mental status, by Moos ${ }^{11)}$, as translated and rearranged into 48 Japanese questions (Table 1) by Kasamatsu et al. ${ }^{12)}$. It was simplified, so as to correspond to the Japanese language, to a six-points scale, ranging from "don't feel at all" at 0 point to "feel acutely" at 6 points. Also, Moos used factor analysis to classify the 48 complaints on the MDQ into the following eight factors, i.e., "pain", "loss of concentration", "behavioral change", "autonomic reaction", "water retention", "negative feelings", "mood uplift", and "control". The subjects respond by placing a mark in the appropriate position of the six segments indicated along a straight line ranging from "don't feel at all" at one end and "feel acutely" at the other.

\section{NASA-TLX}

The NASA-TLX (NASA Task Load Index) was developed by the American National Aeronautics and Space Administration (NASA) and is a scale for subjective evaluation of mental workload ${ }^{13)}$. It is composed of six items: mental demand, physical demand, temporal demand, own performance, effort and frustration level. In this study, scale names and explanations from the Japanese version of the NASA-TLX by Haga and Mizukami ${ }^{14)}$ were used, and the adaptive weighted workload, or AWWL, proposed by Miyake and Kumashiro et al. ${ }^{15)}$, was used for the overall evaluation points. First, evaluation of the six items was done using a visual analogue scale, whereby the subjects placed marks along $12 \mathrm{~cm}$ lines with the extremes of small/large, weak/strong, good/bad, not much/a lot, and low/high at each end, respectively.

\section{Physiological indices \\ Heart rate variability}

The Active Tracer AC-301 was used for the electrocardiograph (ECG). The ECG was fitted to the subjects after they entered the room and were seated, relaxed and breathing normally. The portable ECG was placed on their chests (the positive electrode on the left chest V5, the negative electrode at the highest point of the sternum, and the ground on the right chest opposite the positive electrode) and measurement was done continuously until the end of the 10 min rest phase after task completion. In this study, heart rate variability (HRV) was obtained by autoregressive (AR) analysis of the RR intervals measured $5 \mathrm{~min}$ after the start of the rest phase, before task start, at start, at $10 \mathrm{~min}, 20 \mathrm{~min}, 30 \mathrm{~min}$ and for the first 5 min after completion, and the RR intervals. HRV was at $0.04-0.15 \mathrm{~Hz}$ as low frequency (LF) and at $0.15-0.5 \mathrm{~Hz}$ as high frequency $(\mathrm{HF})$, and the $\mathrm{LF} / \mathrm{HF}$ ratio was used as the index of cardiac sympathetic nerve activity ${ }^{16)}$. Also the autoregressive spectral analysis was performed at a sampling interval of $500 \mathrm{~ms}$, and this was applied to the heartbeat interval data ${ }^{17,18)}$. Although breathing frequency was not controlled in this study, there was no significant difference in the mean breathing frequency, which was shown by the center frequency of respiratory sinus arrhythmia, among time or phase.

\section{Sex steroid hormones}

After completion of the experiment, blood was collected in order to understand sex steroid hormones changes in the three phases of the menstrual cycle. The collected blood was temporarily stored in a refrigerator in the laboratory, and measurements of serum estradiol and progesterone density were carried out using SRL. 
Typically there are three types of estrogen: estrone, estradiol and estriol; of these mainly estradiol is associated with the menstrual cycle.

\section{Measured phases of the menstrual cycle and measurement period}

The subjects were asked to measure and record basal body temperature (BT-14W: $210 \mathrm{~d}$ automatic memory) for three months before the start of the experiment, which was carried out in the follicle phase, luteal phase, and menstruation phase. It was measured in the follicle phase (3-6 d after cessation of bleeding), in the luteal phase (7-10 d before the onset of menstruation), and menstruation phase ( $3 \mathrm{~d}$ after the start of menstruation). Measurement of the basal body temperature was started in June 2006, when subjects reported the menstruation starting date, and the measurement period was adjusted. The experiment was completed within two months; September to October. The basal body temperature was measured until completion of the experiment.

\section{Statistical analysis}

The subjects' follicle, luteal and menstruation phases were measured longitudinally. Dispersion analysis of repeated measurement and Bonferroni's multiple comparison were carried out on the experiment results and serum hormone levels. Two-way layout dispersion analysis and Bonferroni's multiple comparison were performed for HRV. The results of MDQ and NASA-TLX showed significance which were subjected to the Wilcoxon rank sum test, and for all tests $p<0.05$ was the statistical significance level. SPSS14.0j was used for statistical analysis.

\section{Ethical considerations}

This study was examined and approved by the Committee for Research Ethics and Safety of the Oita University of Nursing and Health Sciences.

The subjects participated in the study of their own free will, and it was explained to them both orally and in writing in the study request that they could stop at any time during the experiment, and also that whether or not they participated in the study had absolutely no connection with their grades, and that the data would not be used for any purpose other than the aim of the study. The study request, which the subjects read in advance, explained clearly that the subjects were to measure basal body temperature for three months that the experiment would be carried out in the follicle phase, luteal phase and menstruation phase, and that blood collection would be done, and the subjects' written agreement was obtained.

\section{Results}

Working memory task results in the menstrual cycle

The task results given as working memory index are shown in Table 1. Primary task: working memory task results performed Bonferroni multiple comparison after the repetition measurement analysis of variance as a result of task accomplishment results. Compared to the number of tasks completed in terms of the effect on working memory of three phases of the menstrual cycle: follicle, luteal, and menstruation, it was shown there was no significant difference $(\mathrm{F}(2,22)=2.394, p=0.115)$. The results from mental calculation adding 2 if the patient had a smiley face or subtracting 2 if a tearful face showed a significant difference for the three phases for the task error rate $(\mathrm{F}(2,22)=3.482, p=0.049)$, and that the luteal phase showed a higher significance than the menstruation phase.

For the secondary task that interfered with the primary task, there was no significant difference between phases for the number of correct confirmations of the prescription and error rate.

\section{$M D Q$ scores in the menstrual cycle}

The result of the MDQ is shown in Fig. 2. The number of indefinite complaints was highest in the menstruation phase, followed by the luteal phase, with the fewest complaints occurring in the follicle phase. As a result of the Friedman test, significance was shown for the "pain", "concentration", "behavioral change", "water retention" and "negative feeling" factors. The results of Wilcoxon rank sum test showed that the menstruation phase had sig-

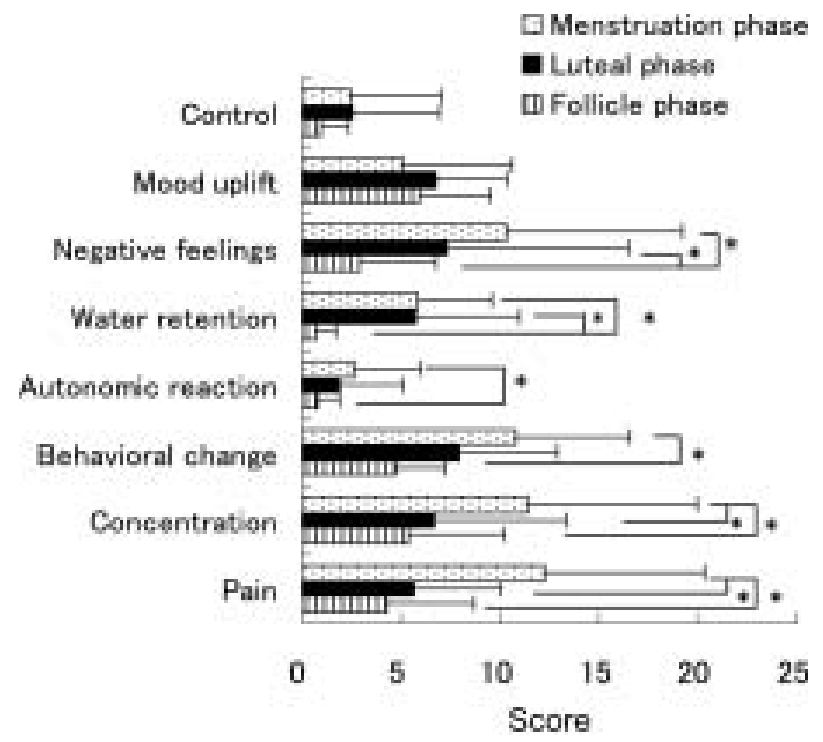

Fig. 2. Menstrual distress questionnaire (MDQ) scores in the menstrual cycle.

$* p<0.05$; Wilcoxon signed-rank test. 


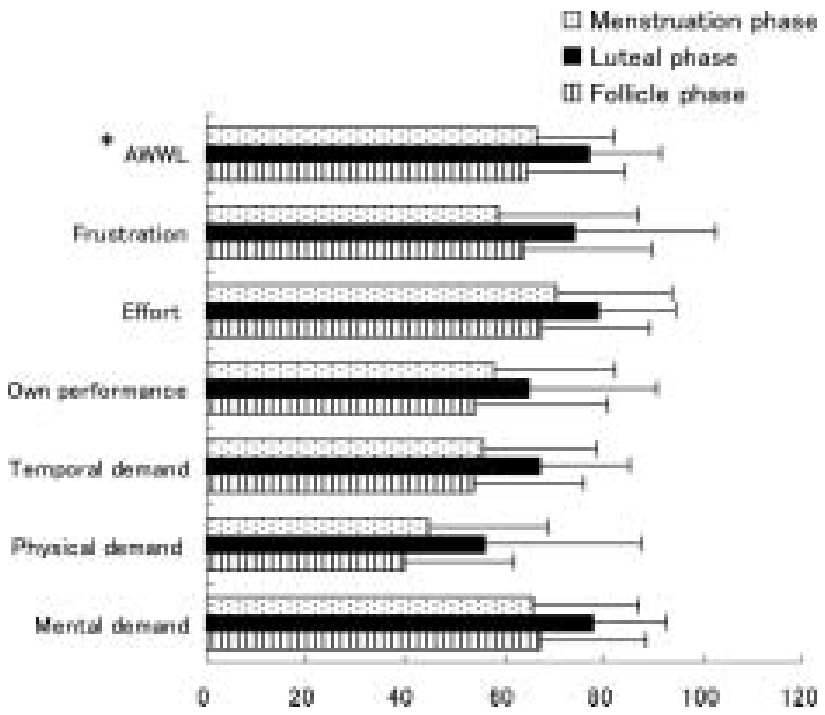

Fig. 3. NASA task load index (NASA-TLX) in the menstrual cycle. *AWWL: Adaptive weighted workload.

nificantly more "pain" factors, such as headache, lumbago, hypogastric pain, and physical listlessness, compared to the follicle phase $(\mathrm{Z}=-2.449, p=0.014)$ and luteal phase $(\mathrm{Z}=-2.313, p=0.021)$. For factors relating to the menstruation phase showed significant reduction in concentration as compared to the luteal phase $(Z=-2.349$, $p=0.019)$ and the follicle phase $(\mathrm{Z}=-2.499, p=0.014)$. For "autonomic reaction" $(\mathrm{Z}=-2.217, p=0.027)$ and "behavioral change" ( $\mathrm{Z}=-2.852, p=0.004)$ factors, complaints were significantly more frequent in the menstruation phase than in the follicle phase. "Water retention" factors were significant in the luteal phases $(Z=-2.317$, $p=0.018)$ and menstruation phases $(\mathrm{Z}=-3.066, p=0.002)$ as compared to the follicle phase. Factors relating to "negative feelings" were significantly more frequent in the menstruation phase $(\mathrm{Z}=-2.807, p=0.005)$ than in the follicle phase. Also, there were significantly many complaints in the luteal phase $(\mathrm{Z}=-2.807, p=0.005)$.

NASA-TLX scores in the menstrual cycle

NASA-TLX (Fig. 3), as for the luteal phase, a mental work load showed a high tendency in "AWWL" which was "an intellectual perception desire" $(\mathrm{Z}=-1.793$, $p=0.073)$, "frustration" ( $\mathrm{Z}=-1.680, p=0.093)$, general evaluation in comparison with the menstruation period $(\mathrm{Z}=-1.67, p=0.099)$, in addition, for "the effort" items $(\mathrm{Z}=-1.655, p=0.098)$, a higher tendency was shown for the luteal phase than for the follicle phase.

\section{Changes in HRV in the menstrual cycle}

The changes in HRV before and after task performance are shown in Fig. 4. By the change of the HF and LF/HF before and after task, three phases of the menstrual cycle
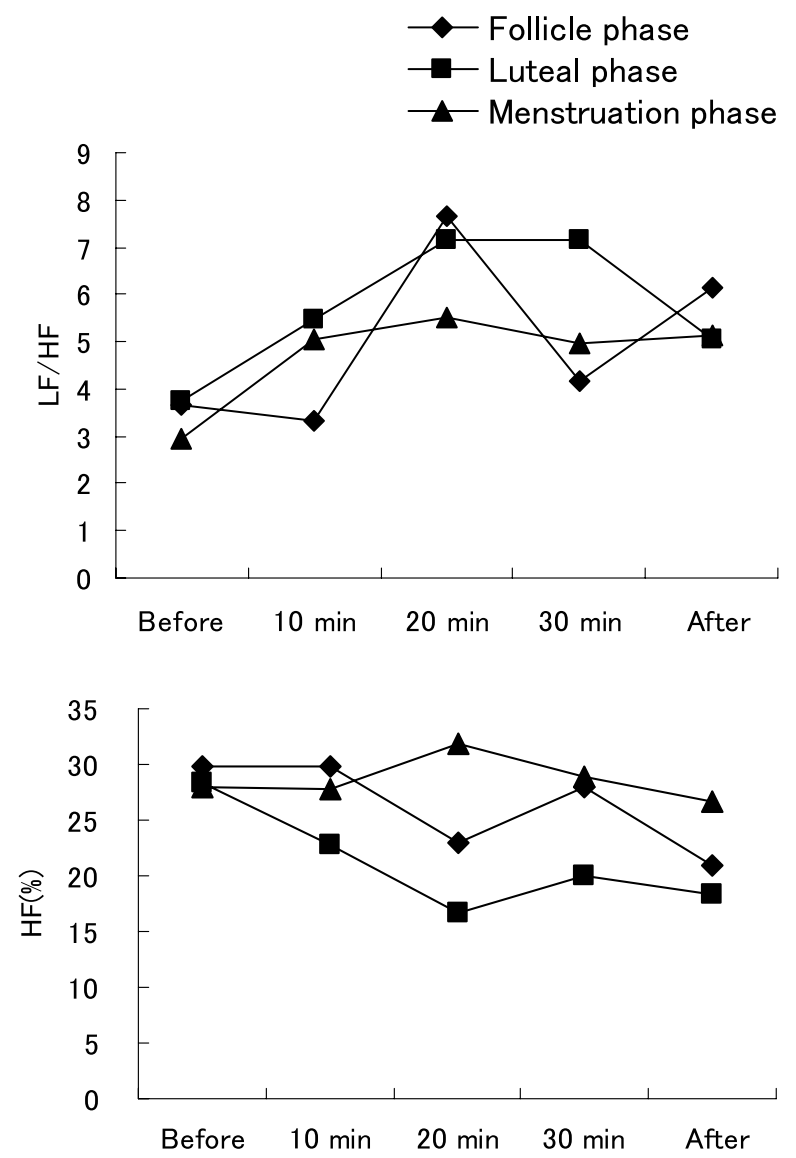

Fig. 4. Changes in HRV before and after task performance $(n=12)$.

Before: Before task performance, Start: After task start, 10 min: $10 \mathrm{~min}$ late, $20 \mathrm{~min}$ : $20 \mathrm{~min}$ late, $30 \mathrm{~min}: 30 \mathrm{~min}$ late, After: After task completion.

did not show a significant difference. However, for all three phases, the LF/HF ratio was a minimum 2.9 before task performance with a maximum 7.7 , showing more than 1 , and indicating that the cardiac sympathetic nerve was predominant.

\section{Sex steroid hormones}

The dynamic phases of sex steroid hormones in the menstrual cycle are shown in Fig. 5. The estradiol values are $70.00 \pm 27.86 \mathrm{pg} / \mathrm{ml}$ for the follicle phase, $172.92 \pm 94.74 \mathrm{pg} / \mathrm{ml}$ for the luteal phase, and $34.42 \pm 7.34 \mathrm{pg} / \mathrm{ml}$ for the menstruation phase. The result of repeated measurement dispersion analysis showed significance in the three phases $(\mathrm{F}(2,22)=20.534, p<0.000)$. The luteal phase showed a significantly high value in comparison with other phases when Bonferroni's multiple comparisons was done. The follicle phase showed a significantly higher value than the follicle phase.

The progesterone values were $0.47 \pm 0.16 \mathrm{ng} / \mathrm{ml}$ for the follicle phase, $12.80 \pm 9.30 \mathrm{ng} / \mathrm{ml}$ for the luteal phase, and 


\section{Estradiol}

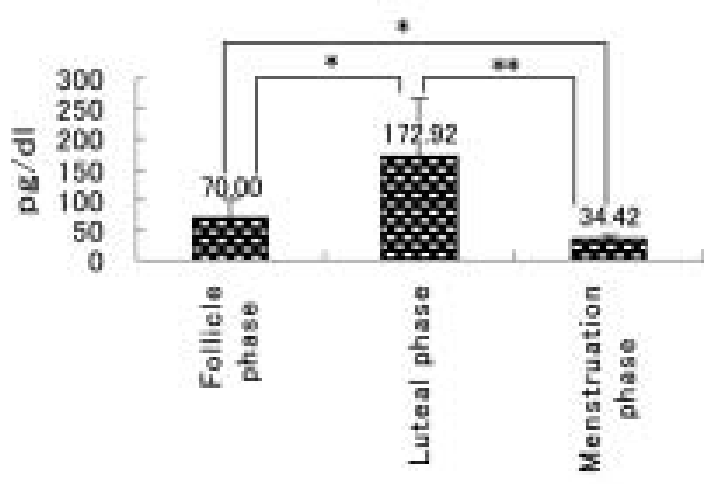

Progesterone

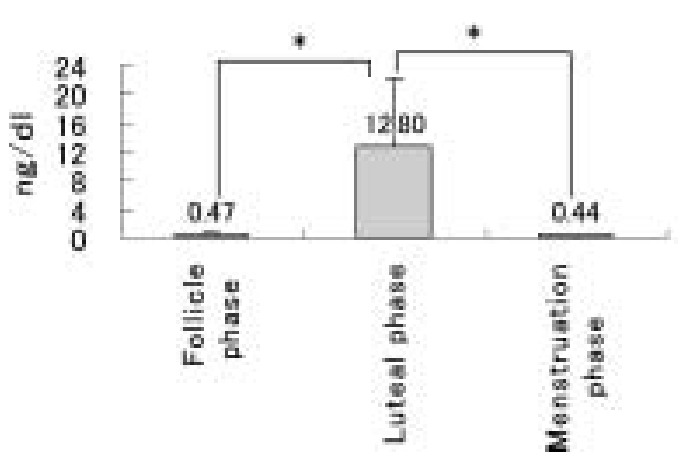

Fig. 5. Sex steroid hormones.

Values are expressed as mean $\pm \mathrm{SE}$.

${ }^{*} p<0.05, * * p<0.01$ : Bonferroni test.

$0.44 \pm 0.14 \mathrm{ng} / \mathrm{ml}$ for the menstruation phase. Repeated measurement dispersion analysis showed significance in the three phases $(\mathrm{F}(2,22)=20.995, p<0.000)$. Compared to the other phases, the luteal phase clearly showed a high value.

\section{Discussion}

For the test tasks a computer was used to program the preparation procedure for the medication administration that is representative of the nursing workplace. The visual information items were added for this program in addition to the previously reported language information items. The test tasks for holding and processing visual and language information were used as the working memory index. These were conducted repeatedly for $30 \mathrm{~min}$. No differences among the three phases were observed in regards to maintaining patient information memory. However, the results considering the mental calculation task where for the patient information 2 was added if the patient is smiling and 2 was subtracted if the patient is tearful, the luteal phase showed a clearly higher error rate than the menstruation phase. These results were converse to the results we reported earlier. In other words, the results did not match the report of the positive relationship between high estrogen quantity and high performance for research relating to language working memory9, 19, 20).

As shown by the research results of Man and Macmillan, et al., there are reports of decline in ability for spatial working memory during the luteal phase, and similar results were found by this study. The tests used for this study were conducted when the luteal phase sex steroid hormone concentration was high. For this reason it is assumed that the difference in task execution ability is due to the affects of the high concentration of sex steroid hormones. According to Shansky et al. ${ }^{21)}$, of the sex steroid hormones, a test regarding estrogen was conducted on female mice to test the relationship between stress and recognition response, and the results showed a correlation between high estrogen levels and stress sensitivity. In other words, the stress sensitivity increases as the amount of estrogen secreted rises, so it is supposed that the level of stress can increase more easily during the luteal phase.

The subjective NASA-TLX index is generally used as a measure for showing the mental workload at the point a task is completed. The results of that study showed a tendency for there to be a strong feeling of "intellectual perception design" and "frustration" during the luteal phase more so than during the menstruation phase and also demonstrated a high tendency for "AWWL," which is a comprehensive evaluation. In other words, this research showed that the mental workload is high during the luteal phase and indicates a feeling of burden during tasks.

On the other hand, the change in HRV did not demonstrate a significant difference during the three phases for the HF value or the LF/HF ratio, but it did show that the $\mathrm{LF} / \mathrm{HF}$ ratio was at least twice as high as the pre-task execution value with the maximum being close to eight times higher, which is a higher ratio than we reported earlier. In other words, it can be supposed that there is psychological tension during task execution and that the sympathetic nerve is ascendant during the three phases.

Next, it has been stated that the regions of the brain activated differs depending on the task characteristics. The region activated by visual working memory is mainly the region in the right hemisphere from the sight region of the occipitis to the sincipita ${ }^{22}$. On the other hand, the region of the brain activated by language working memory, which is a phonological holding task, is mainly the left hemisphere ${ }^{23)}$. In other words, the work of visual and language working memory is presumed to work across a broad region of both the left and right hemispheres of the brain.

From this it is supposed that visual and language work- 
ing memory tasks easily increase stress and mental workload and impact task execution ability during the luteal phase depending on the characteristics of the task.

Let us consider the premenstrual symptoms and task execution ability. In regards to premenstrual symptoms, there are many indefinite complaints during the menstruation phase followed next by the number of such complaints during the luteal phase. For task execution ability, however, the best results were obtained during the menstruation phase. In other words, for visual and language working memory tasks, it was clear that the indefinite complaints had no affect during the menstruation phase.

Furthermore, cognitive neuroscience is proving that working memory is controlled by concentration ${ }^{24}$. Working memory is a mind faculty that carries out multiple tasks simultaneously, in parallel, and involves control of concentration distribution, with individual differences in attention capacity. Further, there is a trade-off between information "retention" and "processing", and because there are capacity restrictions on working memory, when the majority of capacity is used for information processing, only that left over can be used for information retention ${ }^{3,25,26)}$.

This research showed that for the visual and language working memory, during the menstrual cycle the highest error rate was demonstrated during the luteal phase. This error rate was approximately twice that reported earlier by us for language working memory. Possible reasons for this include (1) the differences in task characteristics where the tasks used this time activated a broad region in both the right and left hemispheres of the brain, and (2) the high concentration of sex steroid hormones secreted during the luteal phase increases stress sensitivity.

There is a relationship between medical mistakes made by nurses and insufficient working memory capacity. Measures for alleviating insufficient working memory capacity include having administrators understand the difference in the error occurrence rate during the different phases of the menstrual cycle and reducing the amount of visual and language information. We hope that this study will be utilized as basic knowledge in the area of occupational health and safety for all female workers involved not only in the nursing profession but in all areas such as service industries, medicine, welfare, education and the like.

\section{Conclusion}

This study aimed to examine the effects of follicle phase, luteal phase, and menstruation phase in the menstrual cycle on language and visual working memory. The results suggested that performance is different on the work of the language and visual working memory by the time in the menstrual cycle and that the task performance is worse in the luteal phase. Further, for task loads of $30 \mathrm{~min}$, mental workload was higher in the luteal phase, and stress was more easily felt, indicating predominance of sympathetic nerve activity. Indefinite complaints were most frequent during the menstruation phase, but task performance was good regardless of the indefinite complaint.

\section{Acknowledgements}

We wish to thank the female students (Oita University of Nursing and Health Sciences University) who cooperated with this study.

\section{References}

1) Equal Employment/Children and Families Bureau (2005) 2004 White Paper on Female Labor, Ministry of Health, Labour and Welfare State of Working Women, Foundation, 21st Century Occupation Foundation, 118.

2) Noda Y (2003) Menstrual experiences among junior college students Part 2; Factors affecting to menstrual experiences. J JSPOG 8, 64-78 (in Japanese).

3) Baddeley A (2000) The episodic buffer: a new component of working memory? Trends Cogn Sci 4, 417-23.

4) Osaka N (Ed.) (2000) Brain and Working Memory, 1-5, Kyoto University Press, Kyoto.

5) Konishi K, Kumashiro M, Izumi H, Higuchi Y (2008) Effects of the menstrual cycle on working memory: comparison of postmenstrual and premenstrual phases. Ind Health 46, 253-60.

6) Hampson E (1990) Estrogen-related variations in human spatial and articulatory-motor skills. Psychoneuroendocrinology 15, 97-111.

7) Hampson E, Kimura D (1988) Reciproal effects of hormonal fluctuation on human motor and perceptual skills. Behav Neurosci 102, 456-9.

8) Man MS, Macmillan I, Scott J, Young AH (1999) Mood, neuropsychological function and cognitions in premenstrual dysphoricdisorder. Psychol Med 29, 727-33.

9) Rosenberg L, Park S (2002) Verbal and spatial functions across the menstrual cycle in healthy young women. Psychoneuroendocrinology 27, 835-41.

10) Baddeley A, Hitch G (1974) Working memory. In: The psychology of learning and motivation: advances in research and theory, Vol.8, Bower GH (Ed.), Academic Press, Amsterdam.

11) Moos RH (1968) The development of a menstrual distress questionnaire. Psychosom Med 30, 853-67.

12) Kasamatsu K, Suzuki M, Karashima H, Izumi H, Kumashiro M, Ninomiya R (2004) The effects of the menstrual cycle on the task performance using two kinds of tasks. Ergonomic 40, 125-31 (in Japanese).

13) Hart SG, Staveland LE (1988) Development of NASA- 
TLX, results of empirical and theoretical research. In: Human Mental Workload, Hancock PA, Meshkati N (Eds.), 139-83, Elsevier Science Publishers BV, Amsterdam.

14) Haga S, Mizukami N (1996) Japanese version of NASA task load index: sensitivity of its workload score to difficulty of three different laboratory tasks. Ergonomics 32, 71-9 (in Japanese).

15) Miyake S, Kumashiro M (1993) Subjective mental workload assessment technique -An introduction to NASA-TLX and SWAT and a proposal of simple scoring methods. Ergonomic 29, 399-408 (in Japanese).

16) Aasman J, Mulder G, MulderL J MO(1987) Perator effort and the measurement of heat rate variability. Hum Factors, 29, 161-70.

17) Sato N, Miyake S, Akatsu J, Kumashiro M (1995) Power spectral analysis of heart rate variability in healthy young women during the normal menstrual cycle. Psychosom Med 57, 331-5.

18) Kageyama T, Imai H, Kabuto M (1996) A standardization method for respiratory sinus arrhythmia using breathing frequency (the 2nd report); efficiency of assessing changes in cardiac parasympathetic activity with posture. J Occup Health 38, 107-12.

19) Shaywitz SE, Pugh KR (1999) Effect of estrogen on brain activation patterns in postmenopausal women during working memory tasks. JAMA 281, 1197-202.
20) Grgorova M, Sherwin BB, Tulandi T (2006) Effects of treatment with leuprolide acetate depot on working memory and executive functions in young premenopausal women. Psychoneuroendocrinolog 31, 935-47.

21) Shansky RM, Rubinow $K$, Brennan A, Arnsten AF (2006) The effects of sex and hormonal status on restraint-stress-induced working memory impairment. Behav Brain Funct 7, 2-8.

22) Osaka M, Osaka N, Kondo H, Morishita M, Fukuyama H, Aso T, Shibasaki H (2003) The neural basis of individual differences in working memory capacity; an fMRI study. Neuroimage 18, 789-97.

23) Jonides J, Smith EE, Koeppe RA, Awh E, Minoshima S, Mintun MA (1993) Spatial working memory in humans as revealed by PET. Nature 363, 623-5.

24) Baddeley A, Della Sala S (1996) Working memory and executive control. Phil Trans R Soc Lond B 351, 1397-404.

25) Carpenter PA, Just MA (1989) The role of working memory in language comprehension. In: Basic processes in reading: perception and comprehension, Klahr D and Kotovsky K (Eds.), LEA, Hillsdale.

26) Just MA, Carpenter PA (1992) A capacity theory of comprehension: individual differences in working memory. Psychol Rev 103, 773-80. 